\title{
Urinary glucaric acid excretion in rheumatoid arthritis: influence of disease activity and disease modifying drugs
}

\author{
R Addyman, C Beyeler, C Astbury, H A Bird
}

\begin{abstract}
Objective-To examine if a correlation exists between cytochrome $P-450$ enzyme induction and disease activity in patients with rheumatoid arthritis (RA), measuring urinary excretion of D-glucaric acid (GA) as an index of phase II drug metabolism. Methods-Patients with RA were treated with sulphasalazine, sodium aurothiomalate, or D-penicillamine in standard dose regimens, for 24 weeks. Patients with ankylosing spondylitis (AS) or noninflammatory arthritis (NIA) acted as controls. The urinary GA:creatinine ratio was measured at 0,12 , and 24 weeks of treatment.
\end{abstract}

Results-Patients with RA had a slightly greater urinary GA:creatinine ratio than patients with AS or NIA at baseline; this increased during treatment with disease modifying antirheumatic drugs (DMARDs). Sulphasalazine treatment had a greater effect on GA excretion than sodium aurothiomalate or D-penicillamine; this difference was statistically significant between weeks 0 and $12(p=0 \cdot 01)$. Gamma glutamyltranspeptidase concentration showed a weak correlation with GA excretion between weeks 0 and $12(p=0.03)$, but all other measurements of changes in disease activity (plasma viscosity, C reactive protein, platelets, and articular index) were found not to correlate with GA excretion between weeks 0-12 or 0-24. Conclusion-The increased excretion of GA in patients with RA receiving DMARD treatment is probably the result of an indirect effect on hepatic metabolism bearing no relationship to disease activity.

(Ann Rheum Dis 1996; 55: 478-481)

Rheumatoid arthritis (RA) is the most common inflammatory rheumatic disease of unknown aetiology, characterised by persistent synovitis and complicated by local destruction of bone and cartilage and various systemic manifestations. Its treatment, usually over long periods, comprises compounds providing symptomatic relief (analgesics, nonsteroidal anti-inflammatory drugs (NSAIDs)) and disease modifying anti-rheumatic drugs (DMARDs) with a later onset effect (sodium aurothiomalate, D-penicillamine, sulphasalazine, and others). The aim of treatment is to improve disease activity and to reduce the risk of drug toxicity as much as possible, especially in view of the polypharmacy that is common.

Against the background of the subject's genetic constitution, drug metabolisminvolving different enzyme systems of biotransformation-is affected by many variables such as age, gender, diet, smoking, other environmental factors, and drugs, ${ }^{12}$ and also by hepatic and extrahepatic diseases such as diabetes mellitus, thyroid disease, cardiopulmonary dysfunction, and malignancy. Phase I drug metabolism involves the mixed function oxidase enzyme, cytochrome $P-450$, and is considered to be the principal drug metabolising enzyme system. Located predominantly on the smooth endoplasmic reticulum, it catalyses the oxidation of a wide variety of compounds and it can be induced or inhibited by a wide range of chemically unrelated substances, including drugs. ${ }^{2}$ This is followed by a change in drug efficacy and toxicity, especially for drugs with a small therapeutic ratio and with long term application, necessitating an alteration in the dosage schedule.

States of inflammation also may affect the rate of drug metabolism. In man, it has been shown that fever induced by the steroid pyrogen etiocholanolone or injected endotoxin is followed by impaired hydroxylation of antipyrine, conjugation of bromosulphthalein, and glucuronidation of salicylamide. Acute viral infections and influenza vaccination can impair cytochrome $P-450$ dependent oxidative drug metabolism leading to toxicity of theophylline, phenytoin, and warfarin. In animal models with adjuvant arthritis, a marked impairment of drug metabolism in vitro and in vivo has been shown. ${ }^{3-5}$

There is a need to explore other hepatic metabolic systems including phase II metabolism-which involves the conjugation of oxidised drugs with an ionisable compound, such as glucuronide or sulphate-as these systems may act as markers of disease activity or predict disease response in RA. One such urine marker of phase II metabolism is D-glucaric acid (GA), already shown to be altered in patients with RA. ${ }^{6}$ Sugars, including glucose and galactose, are broken down by a catabolic pathway producing GA from D-glucuronic acid, which can be measured in urine. There is also evidence that this step in the pathway may act as an indicator of enhanced metabolism induced by drugs. Drugs affecting the pathway in either man or animals include phenobarbitone, amidopyrine,

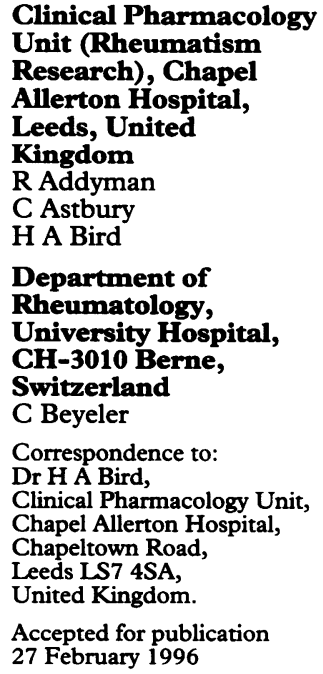

Clinical Pharmacology Unit Chapel Allerton Hospital, Leeds LS7 4SA United Kingdom.

Accepted for publication 27 February 1996 
progesterone, thiopentone, organochloride pesticides, diphenytoin, rifampicin, and the anti-inflammatory, phenylbutazone. ${ }^{78}$

Other factors clearly influence the system. Urinary excretion of GA varies between men and women (53 (SD 10) $\mu \mathrm{mol} / 24 \mathrm{~h}$ and 41 (8) $\mu \mathrm{mol} / 24 \mathrm{~h}$, respectively) though it is not influenced by the menstrual cycle. ${ }^{9}$ Excretion is increased twofold in pregnancy and $3 \cdot 5$-fold in diabetes mellitus, but decreases in neoplastic disease. ${ }^{10}$ Urinary output of GA is also increased in acute viral hepatitis. Fasting reduces excretion twofold within two days, ${ }^{8}$ while chronic cardiac insufficiency, burns, and folic acid status all influence metabolism. There is a correlation between GA and total liver concentration of cytochrome $P-450 .{ }^{11} 12$ Clearly, GA excretion is also influenced by renal function; coefficients of variation as high as $37 \%$ and $27 \%$ have been described. ${ }^{913}$ Adjustment of estimates of GA excretion by expressing it as a proportion of creatinine output in a 24 hour urine sample allows the best option of correcting for incomplete collection and, possibly, renal function. ${ }^{14}$

We have investigated GA excretion in patients with RA treated with three different DMARDs in order to determine the influence, if any, of different drug treatments and the extent to which excretion correlates with conventional indices of rheumatoid disease activity. It also remains a possibility that enhanced GA excretion in RA, if confirmed, may be brought under control with drug treatment and might provide an indication either of hepatic function or of enzyme induction in patients with this disease.

\section{Patients and methods}

Fifty seven patients with RA (12 men and 45 women: age range $29-80$ years (mean 59.8 (SD 12.5) years)) were recruited to the study. Twenty nine patients were recruited to serve as controls: 12 men and one woman with AS (age range 28-55 years (mean $45 \cdot 4(8 \cdot 8)$ years)), and five men and 11 women with NIA (age range 40-71 years (mean 56.6 (10.8) years)).

Patients with RA met the revised criteria of the American Rheumatism Association ${ }^{15}$ and had disease activity sufficient to justify the introduction of DMARDs. They were allocated to treatment with enteric coated sulphasalazine $0.5 \mathrm{~g} /$ day for two weeks, $1 \mathrm{~g} /$ day for two weeks, $1.5 \mathrm{~g} /$ day for four weeks, and 2.0 g/day thereafter), D-penicillamine $(125 \mathrm{mg} /$ day for two weeks, $250 \mathrm{mg} /$ day for two weeks, $375 \mathrm{mg} /$ day for two weeks, and $500 \mathrm{mg} /$ day thereafter), or sodium aurothiomalate by intramuscular injection $(10 \mathrm{mg}$ test dose followed by $50 \mathrm{mg} /$ week for 20 weeks and $50 \mathrm{mg} / \mathrm{month}$ thereafter). Where necessary, because of toxicity, dosage was reduced. NSAIDs and all other treatments were continued at the same dose throughout the 24 week study period wherever possible. Particular attention was paid to those drugs likely to influence the cytochrome $P-450$ system. Where change was necessary on grounds of toxicity, this was carefully documented.
Control subjects comprised patients with ankylosing spondylitis (AS) ( $\mathrm{n}=13)$ (according to the modified New York criteria ${ }^{16}$ ), four of whom were receiving enteric coated sulphasalazine before they entered the study. A second control group comprised patients with non-inflammatory arthritis (NIA) $(n=16)$, characterised by normal plasma viscosity and serum concentration of $C$ reactive protein (CRP), including patients with nonarticular rheumatism $(n=7)$, osteoarthritis $(n=5)$, cervical spondylosis $(n=2)$, low back pain $(n=1)$, and Sudeck's atrophy $(n=1)$, or a combination of these conditions.

Patients attended a special clinic at weeks 0 , 12 , and 24 . A variety of clinical assessments were performed, including those of the Ritchie articular index and early morning stiffness. Haematological assessments included haemoglobin and platelet count, and biochemical assessments of disease activity included plasma viscosity, CRP and gamma glutamyl transpeptidase (GGT) concentrations. At each visit, the patient provided a 24 hour urine specimen for estimation of GA:creatinine ratio.

The volume of each 24 hour urine sample was measured and aliquots frozen and stored at $-20^{\circ} \mathrm{C}$ until required for analysis. Urinary glucaric acid was determined by the method of Simmons et al: ${ }^{16}$ a spectrophotometric assay involving inhibition of $\beta$-glucuronidase/ phenophthalein glucuronide hydrolysis by D-glucaro-1,4-lactone, the latter being the product of urinary GA hydrolysis at $100^{\circ} \mathrm{C}$ and pH 3.6. Urinary creatinine was measured by a standard spectrophotometric assay. The GA: creatinine ratio was calculated (intra-assay precision $3 \cdot 29 \%$, interassay precision $9.98 \%$ ).

Statistical treatment of data included Spearman rank correlation, Wilcoxon signed rank test, Mann-Whitney $U$ test and KruskalWallis analysis of variance as appropriate. All tests were performed using Unistat IV statistical software on a personal computer.

\section{Results}

Among the 57 patients with RA, 19 received sulphasalazine, 16 received D-pencillamine, and 22 received sodium aurothiomalate after adequate periods of washout of other DMARDs. In addition, six of the patients were each receiving concomitant treatment with one of the following potential cytochrome $P-450$ enzyme inducing drugs: hormone replacement therapy, norgestrel, levonorgestrel, tamoxifen, quinalbarbitone, phenylbutazone. Five patients were receiving cimetidine, a known inhibitor of cytochrome $P-450$. Six patients had coronary heart disease and two had diabetes mellitus. GA excretion was neither incresed nor decreased at baseline in any of these patients.

The mean duration of disease activity in patients taking sulphasalazine, D-penicillamine, and gold was $12.9,15.5$, and 9.4 years, respectively (medians 8,15 , and 8.5 years, respectively). Of these three drugs, penicillamine was the one that subsequently showed least effect on GA:creatinine excretion ratio. 


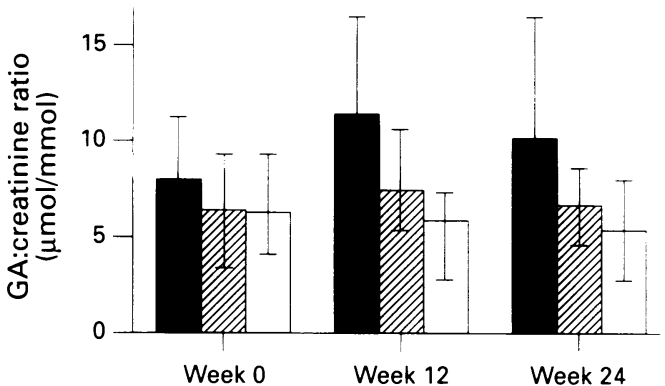

Figure 1 Urinary glucaric acid $(G A)$ :creatinine ratio in patients with rheumatoid arthritis ( $\square$ ), ankylosing spondylitis $(\square)$, or non-inflammatory arthritis $(\square)$ : median and interquartile range.

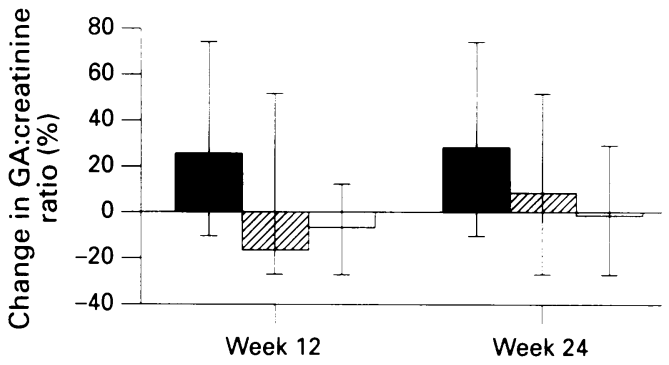

Figure 2 Change in glucaric acid $(G A)$ :creatinine ratio over 24 weeks compared with baseline values, in patients with rheumatoid arthritis ( ), ankylosing spondylitis (घ), or non-inflammatory arthritis ( $\square$ ): median and interquartile range.

At baseline, median GA excretion was 57 $\mu \mathrm{mol} / 24 \mathrm{~h}$ (interquartile range $41-87 \mu \mathrm{mol} / 24$ h) in RA, 68 (34-111) $\mu \mathrm{mol} / 24 \mathrm{~h}$ in AS, and $64(45-83) \mu \mathrm{mol} / 24 \mathrm{~h}$ in NIA (Kruskal-Wallis analysis of variance, $p=0 \cdot 85$ ). Figure 1 shows the median GA excretion corrected for creatinine excretion (GA:creatinine ratio) for all patient groups at weeks 0,12 , and 24 , while figure 2 shows the changes in GA:creatinine ratio over 24 weeks in patients with RA, AS, and NIA.

Patients with $R A$ had a slightly greater urinary GA:creatinine ratio than those with AS or NIA at baseline, though this failed to reach statistical significance (Kruskal-Wallis analysis of variance, $p=0 \cdot 26$ ). During DMARD treatment, the GA:creatinine ratio increased until, at weeks 12 and 24, patients with $\mathrm{RA}$ had a statistically significant greater GA:creatinine ratio than those with AS or NIA (KruskalWallis analysis of variance, $p=0.0003$ and $\mathrm{p}=0 \cdot 002$, respectively).

Effect of disease modifying antirheumatic drug (DMARD) intervention on urinary glucaric acid $(G A)$ :creatinine ratio in patients with rheumatoid arthritis

\begin{tabular}{|c|c|c|c|c|c|}
\hline \multirow[t]{2}{*}{$D M A R D$} & \multicolumn{3}{|c|}{ GA:creatinine ratio ( $\mu \mathrm{mol} / \mathrm{mmol})$} & \multicolumn{2}{|l|}{$t p$} \\
\hline & Week 0 & Week 12 & Week 24 & $\begin{array}{c}\text { Week } 0 \\
\mathrm{v} \\
\text { Week } 12\end{array}$ & $\begin{array}{l}\text { Week } 0 \\
\mathrm{v} \\
\text { Week } 24\end{array}$ \\
\hline Sulphasalazine & $\begin{array}{l}7 \cdot 1 \\
(5 \cdot 8-14 \cdot 6) \\
(n=19)\end{array}$ & $\begin{array}{l}14 \cdot 3 \\
(11 \cdot 3-16 \cdot 2) \\
(n=15)\end{array}$ & $\begin{array}{l}14 \cdot 6 \\
(6 \cdot 8-21 \cdot 2) \\
(n=15)\end{array}$ & $0 \cdot 061$ & $0 \cdot 125$ \\
\hline Gold & $\begin{array}{l}7 \cdot 3 \\
(5 \cdot 4-11 \cdot 4) \\
(n=22)\end{array}$ & $\begin{array}{l}11 \cdot 0 \\
(8 \cdot 9-16 \cdot 6) \\
(n=20)\end{array}$ & $\begin{array}{l}10 \cdot 2 \\
(7 \cdot 7-14.0) \\
(n=19)\end{array}$ & $0 \cdot 040$ & $0 \cdot 314$ \\
\hline D-Penicillamine & $\begin{array}{l}9 \cdot 2 \\
(6 \cdot 2-15 \cdot 5) \\
(n=16)\end{array}$ & $\begin{array}{l}9 \cdot 9 \\
(6 \cdot 3-16 \cdot 4) \\
(n=14)\end{array}$ & $\begin{array}{l}9 \cdot 7 \\
(8 \cdot 1-16 \cdot 1) \\
(n=12)\end{array}$ & $0 \cdot 638$ & 0.071 \\
\hline$\neq \mathrm{p}$ & $0 \cdot 3648$ & $0 \cdot 2125$ & 0.5198 & & \\
\hline
\end{tabular}

Values are median (interquartile range)

†Wilcoxon signed rank test; $\ddagger$ Kruskal-Wallis analysis of variance.
The same trends were seen when the data were analysed in two groups according to gender, though the median GA:creatinine ratio was slightly smaller in men $(6.5(4 \cdot 0-8 \cdot 1)$ $\mu \mathrm{mol} / \mathrm{mmol})$ than in women $(8.8(5.8-14.6)$ $\mu \mathrm{mol} / \mathrm{mmol}$ ) at baseline.

The GA:creatinine ratio plotted against age showed good distribution in both RA and control groups, with no correlation between age and GA:creatinine ratio in either group (Spearman rank correlation $\mathrm{p}=0.34$ and $\mathrm{p}=0 \cdot 21$, respectively).

Patients with RA who smoked had slightly smaller GA:creatinine ratios compared with nonsmokers, though this did not reach statistical significance (Mann-Whitney $U$ test, $\mathrm{p}=0.32$ ).

Table 1 shows the effect of DMARD intervention on GA:creatinine ratio in patients with RA. There was a difference according to the drug used to obtain remission. For sulphasalazine, the GA:creatinine ratio increased consistently between weeks 0 and 12 as a response was obtained, and it remained increased thereafter. There was a more modest increase in groups of patients treated with sodium aurothiomalate and D-pencillamine, the maximum change again occurring between weeks 0 and 12 , at the time when clinical improvement was usually most pronounced.

An expected decrease in plasma viscosity was observed in the patients with RA over the 24 week period: from $1.83(1.72-1.94) \mathrm{mPa}$ at week 0 , decreasing to $1.75(1.69-1.89) \mathrm{mPa}$ and to $1.74(1.64-1.83) \mathrm{mPa}$ at weeks 12 and 24, respectively (Wilcoxon signed rank test: $p=0.003$ and $p=0.0003$, respectively). Early morning stiffness and articular index, the only other two clinical parameters measured, both decreased with disease modifying intervention, but this was not correlated with changes with changes in GA excretion.

Median GGT activity was 20 (14-33) U/1 at baseline and increased slightly at week 12 , to 22 (12-32) U/l, followed by a statistically significant decrease from baseline, to 19 (12-29) $\mathrm{U} / 1$ at week 24 (Wilcoxon signed rank test, $\mathrm{p}=0.01)$. There was a weak correlation between changes in GA:creatinine ratio and changes in GGT for weeks (0-12) (Spearman rank correlation, $p=0.03$ ).

Clinical, biochemical, and haematological markers of disease activity all showed a decrease upon DMARD intervention, but these decreases did not correlate with changes in the GA:creatinine ratio.

\section{Discussion}

The aim of this study was to investigate whether a correlation exists between cytochrome $P-450$ enzyme induction and disease activity in patients suffering from RA, using patients with $A S$ as a secondary inflammatory polyarthritis and patients with NIA as control groups. Urinary GA was measured as an index for phase II drug metabolism.

Analysis of the results for those potentially confounding patients who were receiving cytochrome $P-450$ enzyme inducing drugs (quinalbarbitone, levonorgestrel, tamoxifen, 
and phenylbutazone) and enzyme inhibiting drugs (cimetidine) revealed no increase or decrease in the GA:creatinine ratio at week 0 . Statistically significant findings were thought not to result from inaccuracies in the assay technique, as the precision of the assay was very good. Even though a large between patient variability was seen, the median baseline GA excretion values were well matched between patient groups and were within the ranges previously published ${ }^{17}$. It is well established that increased GA excretion caused by foreign compounds nearly always indirectly accompanies an enhancement of cytochrome $P-450$ reactions. However, there is no evidence in the literature that any of the DMARDs used here for patient treatment induces or inhibits any of the cytochrome $P-450$ isoenzymes. Increased GA excretion was therefore unlikely to be caused by direct induction of cytochrome $P-450$ as a result of DMARD treatment.

The weak correlation seen with changes in GGT and GA:creatinine ratio between week 0 and week 12 may not be of practical significance. However, the non-significance of correlation between other markers of disease activity and change in GA excretion indicates that the increase in GA excretion between weeks 0 and 12 is unlikely to reflect an improvement in disease activity, but is more possibly the result of increased production of glucuronate via the degradation of haemoglobin to urobilinogen, because of increased liver metabolism. We conclude, therefore, that glucaric acid excretion cannot be used as a satisfactory index for disease activity in RA.

We thank the Swiss National Foundation and the Arthritis and Rheumatism Council (B0132) for financial support. We also thank Mrs J L Childs for secretarial help.
1 Park B K, Breckenridge A M. Clinical implications of enzyme induction and enzyme inhibition. Clin Pharmacokinet 1981; 6: 1-24.

2 Küpfer A, Preisig R. Inherited defects of hepatic drug metabolism. Semin Liver Dis 1983; 3: 314-54.

3 Morton D M, Chatfield D $H$. The effects of adjuvant induced arthritis on the liver metabolism of drugs in rats. Biochem Pharmacol 1970; 19: 473-81.

4 Beck F J, Whitehouse $M$ W. Effect of adjuvant disease in rats on cyclophosphamide and isophosphamide metabolism. Biochem Pharmacol 1973; 22: 2453-68.

5 Cawthorne M A, Palmer E D, Green J. Adjuvant-induced arthritis and drug metabolizing enzymes. Biochem Pharmacol 1976; 25: 2683-8.

6 McKenna F, Coombes G, Sandle L, Mather H. D-Glucaric acid in rheumatoid arthritis [abstract]. $\mathrm{Br} \mathcal{F}$ Rheumatol 1992; 31 (suppl 2): 115.

7 Aarts E M. Evidence for the function of D-glucarcid acid as an indicator for drug induced enhanced metabolism an indicator for drug induced enhanced metabolism through the glucuronic acid

8 Sotaumi E A, Medzihradsky F, Eliasson G. Glucaric acid as an indicator of use of enzyme-inducing drugs. Clin Pharmacol Ther 1974; 15: 417-23.

9 Mowat A P. Developmental effects of liver D-glucuronolactone dehydrogenase levels on D-glucaric acid excretion in urine; hormal effects on D-glucaric acid excretion in urine. $\mathcal{F}$ Endocrinol 1968; 42: 585-90.

10 Marsh C A, Carr J. Changes in enzyme activity related to D-glucaric acid synthesis with age, pregnancy and malignancy. Clin Sci 1965; 28: 209-17.

11 Hunter J, Maxwell J D, Stewart D A, Williams R. Urinary D-glucaric acid excretion and total liver content of cytochrome P450 in guinea pigs: relationship during cytochrome P450 in guinea pigs: relationship during
enzyme induction and following inhibition of protein enzyme induction and following inhibition
synthesis. Biochem Pharmacol 1973; 22: 743-7.

12 Lecamwasam D S, Franklin C, Turner P. Effect of phenobarbitone on hepatic drug-metabolising enzymes and urinary D-glucaric acid excretion in man. $\mathrm{Br} \mathcal{F}$ Clin Pharmacol 1975; 2: 257-62.

13 Gilbert J C, Scott A K, Galloway D B, Petric J C Ethosyximide: liver enzyme induction and D-glucaric acid excretion. Br 7 Clin Pharmacol 1974; 1: 249-52.

14 Hunter J, Maxwell J D, Carrella M, Stewart D A, Williams R. D-glucaric acid excretion as a test for hepatic enzyme induction in man. Lancet 1971 ; i: 572-5.

15 Amett F C, Edworthy S M, Bloch D, et al. The American Rheumatism Association 1987 revised criteria for the classification of rheumatoid arthritis. Arthritis Rheum 1988; 31: 315-24.

16 van der Linden S, Valkenburg H A, Cats A. Evaluation of diagnostic criteria for ankylosing spondylitis. A proposal for modification of the New York criteria. Arthritis Rheum 1984; 27: 361-8.

17 Simmons C J, Davis M, Dordoni B, Williams R. Urinary D-glucaric acid assay by an improved enzymatic procedure. Clin Chim Acta 1974; 51: 47-51. 